\title{
HVAC Model Based Fault Detection by Incremental Online Support Vector Machine
}

\author{
Davood Dehestani
}

\begin{abstract}
Preventive maintenance plays an important role in Heating, Ventilation and Air Conditioning (HVAC) system. One cost effective strategy is the development of analytic fault detection and isolation (FDI) module by online monitoring the key variables of HAVC systems. This paper investigates real-time FDI for HAVC system by using online Support Vector Machine (SVM), by which we are able to train a FDI system with manageable complexity under real time working conditions. It is also proposed a new approach which allows us to detect unknown faults and updating the classifier by using these previously unknown faults. Based on the proposed approach, a semi unsupervised fault detection methodology has been developed for HVAC systems
\end{abstract}

Index Terms-Intelligent method, unsupervised fault detection, online SVM, HVAC system.

\section{INTRODUCTION}

There are not many energy systems so commonly used in both industry and domestic as HVAC systems. Moreover, HVAC system usually consumes the largest portion of energy in building for both in industry and domestic. It is reported [1] air-conditioning of buildings accounts for $28 \%$ of the total energy end use of commercial sectors. From $15 \%$ to $30 \%$ of the energy waste in commercial buildings is due to the performance degradation, improper control strategy and malfunctions of HVAC systems. Regular check and maintenance are usually the keys for reaching these goals. However, due to the high cost of maintenance, preventive maintenance plays an important role. A cost effective strategy is the development of fault detection and isolation (FDI).

Several strategies have been employed as a FDI modular in a HVAC system. These strategies can be mainly classified in two categories: model based strategy and signal processing based strategy [2]-[4]. Model-based techniques either use mathematical model or a knowledge model to detect and isolate the faulty modes. These techniques include but not limited to observer-based approach[5], parity-space approach[6], and parameter identification based methods[7]. Henao[8] reviewed fault detection based on signal processing. This procedure involves mathematical or statistical operations which are directly performed on the measurements to extract the features of faults. Intelligent methods such as Genetic Algorithm (GA), Neural Network (NN) and Fuzzy Logic had been applied during last decade for fault detection. Neural network has been used in the

Manuscript received November 2, 2012; revised December 26, 2012

Davood Dehestani is with the University of Technology Sydney (UTS), Building1, UTS building, Broadway, Sydney Australia (e-mail: Davood.dehestani@student.uts.edu.au). variety range of systems for fault detection even for HVAC systems [9]. Lo [10] proposed intelligent technique based on fuzzy-genetic algorithm (FGA) for automatically detecting faults on HVAC systems. However, many intelligent methods such as NN often require big data set for training. Some of them are not fast enough to realize real time fault detection and isolation. This paper investigates methods with real time operation capability and requiring less data. Support Vector Machine (SVM) has been extensively studied in data mining and machine learning communities for the last two. SVM is capable of both classification and regression. It is easy to formulate a fault detection and isolation problem as a classification problem.

SVM can be treated as a special neural network. In fact, a SVM model is equivalent to a two-layer, perceptron neural network. With using a kernel function, SVM is an alternative training method for multi-layer perceptron classifiers in which the weights of the network are identified by solving a quadratic programming problem under linear constraints, rather than by solving a non-convex unconstrained minimization problem as in standard neural network training. Liang[2] studied FDI for HVAC systems by using standard SVM (off-line). In this chaper, incremental SVM (on-line) has been applied. It is required to solve a quadratic programming (QP) for the training of a SVM. However, standard numerical techniques for QP is infeasible for very large datasets which is the situation for fault deteciton and isolation for HVAC systems. By using online SVM, the large-scale classification problems can be implemented in real time configuration under limited hardware and software resources. Furthermore, this paper also provided a potential approach for the implementation of FDI under unsupervised learning frame work.

Based on the model structure given in paper [2], we constructed a HVAC model by using Matlab/Simulink and identified the variables which are more sensitive to commonly encountered HVAC faults. Finally, the effectiveness of the proposed online FDI approach has been verified and illustrated by using Simulink Simulation Platform. The chapter is organized as follows. Section II provides the details about HVAC system modelling. In Section III, the proposed online SVM based FDI approach is presented, which is followed by the simulation studies in Section IV. Conclusions are drawn in Section V.

\section{HVAC SYSTEM MODELLING}

\section{A. HVAC System}

In parallel to the modelling of other energy systems, HVAC modelling was developed by Arguello-Serran[11], Bourhan Tashtoush[12] and others. Gian Liang [13] developed a dynamic model of the HVAC system with single 
zone thermal space. A model of HVAC for thermal comfort control based on NN was also developed by Liang [13]. Based on these models [13], Liang developed a new model for fault detection and diagnosis [2].

Specifically, a few changes in control signal and model parameters have been made in order to well match real applications. Fig. 1 shows a simple schematic of a HVAC system. It consists of three main parts: Air Handling Unit (AHU), the chillier and the control system. When HVAC system starts to work, fresh air passes from a heat exchanger cooling coil section to change heat between fresh air and cooling water. Cooled fresh air is forced by a supply fan to the room.

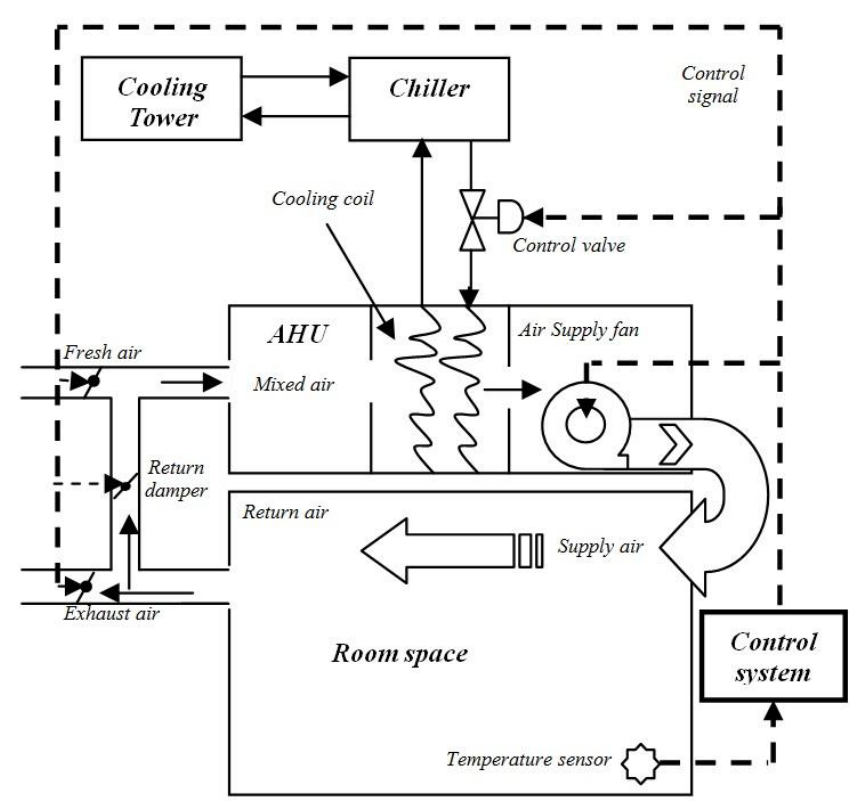

Fig. 1. General schematic of a HVAC system.

After just a few minutes, the return damper opens to allow room air come back to AHU. Then the mixing air passes from cooling coil section to decrease its temperature and humidity. A trade off among exhaust, fresh and return air is decided by control unit. Also the temperature of the room is regulated by adjusting the flow rate of cooling water by a certain control valve. Fig. 2 shows the block diagram of the HVAC system with a simple PI controller. The model is consisting of eight variables in which six variables define as states variables.

Two pressure (air supply pressure $P_{s}$ and room air pressure $P_{a}$ ) and four temperature (wall temperature $T_{w}$, cooling coil temperature $T_{c c}$, air supply temperature $T_{s}$, and room air temperature $T_{a}$ ) are considered as six states. Cooling water flow rate $f_{c c}$ is considered as control signal. Also all six mentioned states variables with cooling water outlet temperature $T_{\text {water_out }}$ is considered as system outputs. But just one of them (room temperature $T_{a}$ ) acts as feedback signa. It should be noted that $T_{\text {-water out }}$ does not use as a state variable in this modelling and it is just used as auxiliary parameter for finding faults.

The states, control input, and controlled output are listed as follows:

$$
\begin{gathered}
X=\left[P_{a} ; P_{s} ; T_{w} ; T_{c c} ; T_{s} ; T_{a}\right], \quad U=\left[f_{c c}\right] \\
Y=\left[P_{a} ; P_{s} ; T_{w} ; T_{c c} ; T_{s} ; T_{a}\right]
\end{gathered}
$$

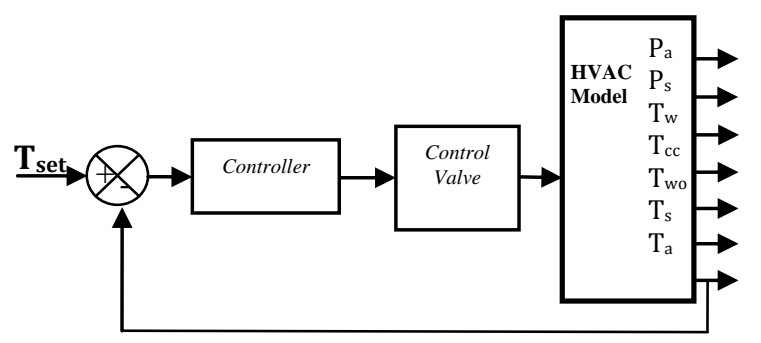

Fig. 2. Block diagram of HVAC model with PI controller.

For the control of HVAC system, the most popular method is Proportion-Integration (PI) control with the flow rate $f_{c c}$ served as the control input. In this study, we therefore select PI controller, and tune the controller by using Ziegler-Nichols method (Reaction Curve Method).

In order to simulate the environmental disturbance in real application, two disturbances are considered in the model: outdoor temperature and outdoor heating (or cooling) load. Outdoor heat/cooling loading will disturb the system but it cannot be measured directly. Though it can be estimated based on the supply/return air temperature/humidity via a load observer [11], for convenience, it is assumed the two disturbances are sinusoidal functions.

\section{B. Parameter Setting}

Table 1 show some major parameters used in this simulation. Without loss of generality, simulation runs in cooling mode. It is easy to consider heating mode just by changing some parameters in table 1 . Range of disturbances are $24-30{ }^{\circ} \mathrm{C}$ and $0.8-1 \mathrm{~kW}$ for outside temperature and heat loading respectively. Mixed air ratio for this model is constant value when system is working properly. The inlet water temperature is set as $\mathrm{T}_{\text {water in }}=7{ }^{\circ} \mathrm{C}$ whilst the outlet water temperature is set as $\mathrm{T}_{\text {water_out }}=9{ }^{\circ} \mathrm{C}$ which may be disturbed by the cooling load.

Same as intelligent systems, the parameters of online SVM classifier should be trained first. First parameter is maximum penalty. By define the maximum penalty of 10 we could achieved best margin for SVM. Based on the testing of different kernel functions, Gaussian function chooses as the best kernel function with $\sigma=1.25$.

TABLE I: MAJOR PARAMETER SET FOR SIMULATION

\begin{tabular}{ll}
\hline \hline Parameter definition & Setting value \\
\hline Temperature set point & $27.5^{\circ} \mathrm{C}$ \\
Room space dimension & $5 \mathrm{~m} \times 5 \mathrm{~m} \times 3 \mathrm{~m}$ \\
Indoor cooling load range & $0.8-1 \mathrm{~kW}$ \\
Outdoor temperature range & $24-30{ }^{\circ} \mathrm{C}$ \\
Outdoor humidity & $55-75 \%$ \\
Max chilled water flow rate & $0.5 \mathrm{~kg} / \mathrm{s}$ \\
Air flow rate & $980 \mathrm{~m} 3 / \mathrm{h}$ \\
Mixed air ratio & 4 \\
Noise of temperature & $5 \% \mathrm{mean}$ value \\
Air handling unit volume & $2 \mathrm{~m} \times 1 \mathrm{~m} \times 1 \mathrm{~m}$ \\
Out side pressure & $1 \mathrm{~atm}$. \\
Inlet water temperature & $7{ }^{\circ} \mathrm{C}$ \\
&
\end{tabular}

\section{Simulation}

All initial values of temperature are set at the morning time. Simulation lasts for 12 hours (from 8:00 am to 8:00 pm). The output temperature for normal condition (with out fault) is shown in Fig 3. 


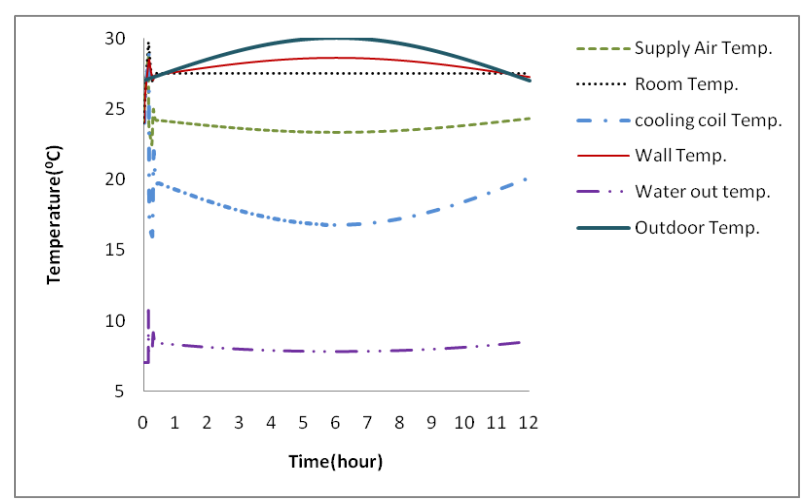

Fig. 3. Temperatures in normal condition of HVAC.

The first half hour of simulation is the transient of system. Room temperature could stay in the desired value (set point) but other temperatures change with noise profile due to their effort for adjusting the room temperature with the set point. Wall temperature is function of outside temperature as it follows outdoor temperature profile. But other internal temperatures follow an inverse profile of outdoor temperature.

Fig. 4 shows the control signal during simulation time.

This flow rate is controlled by a control valve generated from the PI controller.

Cooling water flow rate reaches its maximum value at noon due to highest value of outlet heat and temperature at this time. Some fluctuation in control signal and other variables at beginning time of simulation is related to transient behaviours of the system.

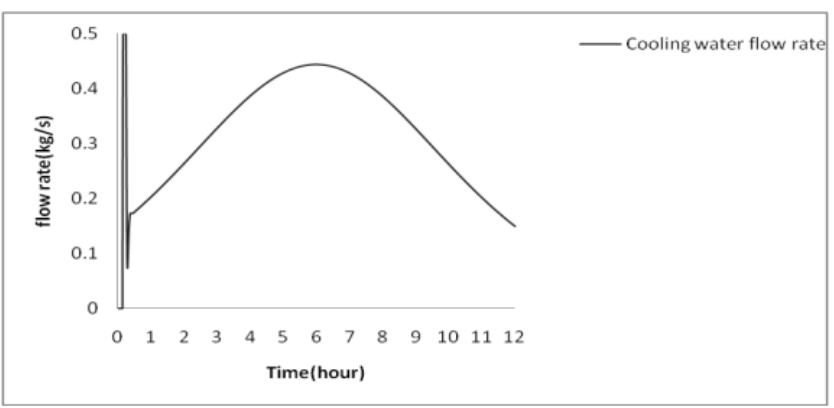

Fig. 4. water flow rate as the function of control signal.

\section{Fault Introduction}

HVAC system may suffer from many faults or malfunctions during operation. Three commonly encountered faults are defined in this simulation:

- Supply fan fault

- Return damper fault

- Cooling coil pipes fouling

Usage of these faults is just to test the performance of proposed fault detector system. Incipient faults applied to test the proposed fault detector due to its difficulty to detection. Four different models consist of one healthy model and three faulty models are generated.

Fig. 5 shows general profile of the fault during one day. The amplitude of the faults gradually increased for 6 hours to reach their maximum values then they stay in this state for 4 hours and returns gradually to normal condition during the last 6 hours. This fault profile has been applied for each fault with some minor changes. In air supply fan fault this profile is used with gain of 10 .

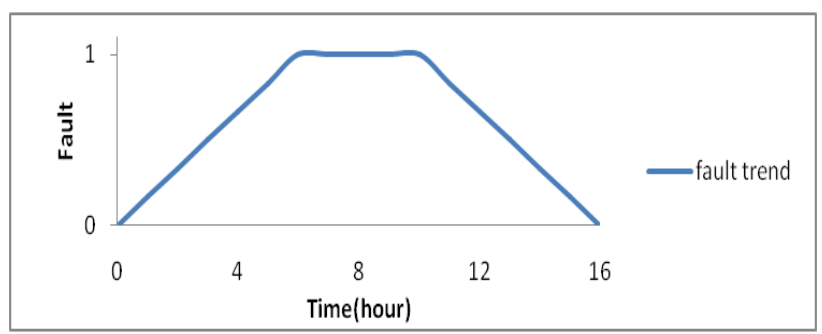

Fig. 5. Fault trend during one day.

In damper fault it is used with gain of -2 and shift point of +4 . For pipe fault it is used with gain -0.3 and shift point of +1 . The most sensitive parameters have been identified for each fault. For the air supply fan fault the major most sensitive parameter is the air supply pressure that changes between 0 to 10 Pascal during fault period. The mixed air ratio is selected as the indicator of the damper fault that decreases from 4 to 2 . Cooling water flow rate decrease from $\mathrm{f}_{\mathrm{cc}}$ to $0.7 \mathrm{f}_{\mathrm{cc}}$ in cooling coil tube fault.

\section{E. Parameter Sensitivity}

The sensitivity of variables respect each fault is analysed in this subsection. Fig. 6 shows sensitivity of cooling water flow rate respect to different faulty modes. It is sensitive to all three faults (damper fault, supply fan fault and cooling water tube fault) with sensitivity of $0.03 \mathrm{~kg} / \mathrm{s}, 0.07 \mathrm{~kg} / \mathrm{s}$ and 0.08 $\mathrm{kg} / \mathrm{s}$.

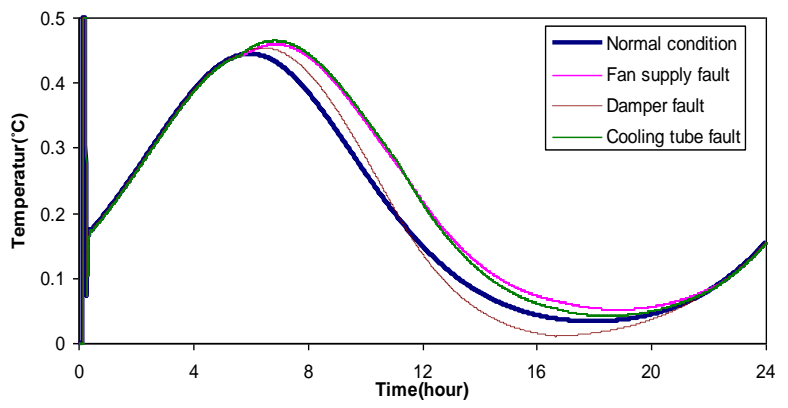

Fig. 6. Cooling water flow rate changes.

Other analysis shows cooling coil temperature and outlet water temperature are sensitive to both supply fan fault and return damper fault. Based on this sensitivity analysis, six parameters consisting of air supply, room pressure, air supply temperature, cooling coil temperature, outlet water temperature, and water flow rate are used for training of supply fan fault. Also three parameters consist of cooling coil temperature, outlet water temperature and water flow rate are applied for training of return damper fault but just water flow rate used for training of third fault (Cooling coil pipes fouling).

\section{ONLINE SUPPORT VECTOR ClASSIFICATION}

\section{A. Incremental-Decremental Online SVM}

The main advantages of SVM include the usage of kernel trick (no need to know the non-linear mapping function), the global optimal solution (quadratic problem), and the generalization capability obtained by optimizing the margin [14]. However, for very large datasets, standard numeric techniques for QP become infeasible. An on-line alternative, that formulates the (exact) solution for $\ell+1$ training data in 
terms of that for $\ell$ data and one new data point, is presented in online incremental method. Training an SVM incrementally on new data by discarding all previous data except their support vectors, gives only approximate results [16]. Cauwenberghs[15] consider incremental learning as an exact on-line method to construct the solution recursively, one point at a time. The key is to retain the Kuhn-Tucker (KT) conditions on all previous data, while adiabatically adding a new data point to the solution. Leave-one-out is a standard procedure in predicting the generalization power of a trained classifier, both from a theoretical and empirical perspective [17].

Giving $\mathrm{n}$ data, $S=\left\{x_{i}, y_{i}\right\}$ and $y_{i} \in\{-1,+1\}$ where $\mathrm{x}_{\mathrm{i}}$ represents the condition attributes, $y_{i}$ is the class label (correct label is +1 and faulty label is -1 ), and $i$ is the number of data for train. The decision hyperplane of SVM can be defined as $(w, b)$, where $w$ is a weight vector and $b$ a bias. Let $\mathrm{w}_{0}$ and $\mathrm{b}_{0}$ denote the optimal values of the weight vector and bias. Correspondingly, the optimal hyperplane can be written as:

$$
w_{0}^{T}+b_{0}=0
$$

To find the optimum values of $w$ and $b$, it is required to solve the following optimization problem:

$$
\min _{w, b, \xi} \quad \frac{1}{2} w^{T} w+C \sum_{i} \xi_{i}
$$

Subject to $\quad y_{i}\left(w^{T} \varphi\left(x_{i}\right)+b\right) \geq 1-\xi_{i}$

where $\xi$ is the slack variable, $\mathrm{C}$ is the user-specified penalty parameter of the error term $(\mathrm{C}>0)$, and $\varphi$ is the kernel function. SVM can change the original non-linear separation problem into a linear separation case by mapping input vector on to a higher feature space. On the feature space, the two-class separation problem is reduced to find the optimal hyperplane that linearly separates the two classes transformed in to a quadratic optimization problem. Depend on problem type, several kernel functions are used.

Two best kernel functions for classification problems are Radial Basis Function (RBF) and Gaussian function regard to nonlinearity consideration.

$$
K\left(x_{i}, x_{j}\right)=\exp \left\{-\gamma\left\|x_{i}-x_{j}\right\|^{2}\right\}, \gamma>0 \quad R B F
$$

In SVM classification, the optimal separating function reduces to a linear combination of kernels on the training data, $f(x)=\sum_{j} \alpha_{j} y_{j} K\left(x_{j}, x\right)+b$,with training vectors $\boldsymbol{x}_{i}$ and corresponding labels $\boldsymbol{y}_{i}$ in the dual formulation of the training problem, the coefficients $\boldsymbol{\alpha}_{i}$ are obtained by minimizing a convex quadratic objective function under constraints.

$$
\min _{0<\alpha_{i}<C} \quad W=1 / 2 \sum_{i j} \alpha_{i} Q_{i j} \alpha_{j}-\sum_{i} \alpha_{i}+b \sum_{i} y_{i} \alpha_{i}
$$

With Lagrange multiplier (and offset) $b$, and the symmetric positive definite kernel matrix $Q_{i j}=y_{i} y_{j} K\left(x_{i}, x_{j}\right)$ the first-order conditions on $W$ reduce to the Kuhn-Tucker (KT) condition:

$$
\frac{\partial W}{\partial \alpha_{i}}\left\{\begin{array}{rr}
>0 ; & \alpha_{i}=0 \\
=0 ; & 0<\alpha_{i}<C \\
<0 ; & \alpha_{i}=C
\end{array} \quad \frac{\partial W}{\partial b}=0\right.
$$

The margin vector coefficients change value during each incremental step to keep all elements in equilibrium. i.e., keep their KT conditions satisfied. It is naturally implemented by decremental unlearning, adiabatic reversal of incremental learning, on each of the training data from the full trained solution. Incremental learning and, in particular, decremental unlearning offer a simple and computationally efficient scheme for on-line SVM training. It has also exact leave-one-out evaluation of the generalization performance on the training data.

\section{B. Algorithm}

There are huge amounts of data generated before a fault happens as most HVAC system is rather reliable. For large datasets, standard SVM techniques (off line SVM) become infeasible.

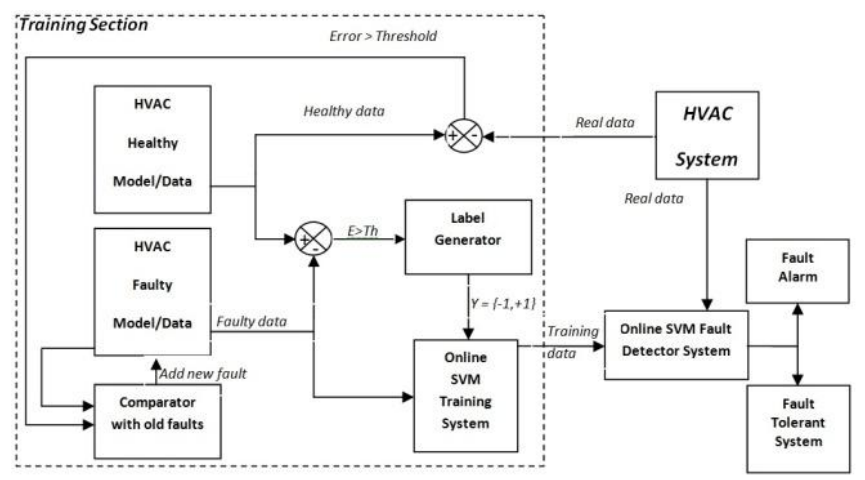

Fig. 7. Schematic of semi unsupervised fault detection.

This motivates the usage of incremental-decremental SVM (online SVM). Fig. 7 shows the proposed fault detection scheme by using incremental-decremental support vector machine classification. The main purpose of the system is to detect un-known faults by monitoring key HVAC variables as discussed in Subsection II.D during system operation.

In this algorithm new faults can be detect (as unknown new faults) by comparing with the outputs of the healthy model and the real system. If detected fault was similar to old fault, it will be categorized by algorithm as existing faults. Otherwise, this data is sent to online SVM trainer for training for the new fault. Finally new fault will be isolate by this online SVM as a known fault. The incremental procedure is reversible and decremental unlearning of each training sample produces an exact leave-one-out estimate of faults with using all HVAC data during its operating. The main advantage of this algorithm is usage of only a range of useful data (including healthy data, old faults, and new faults) instead of whole data sets. Based on this online training procedure, semi-unsupervised fault detection can be implemented. 


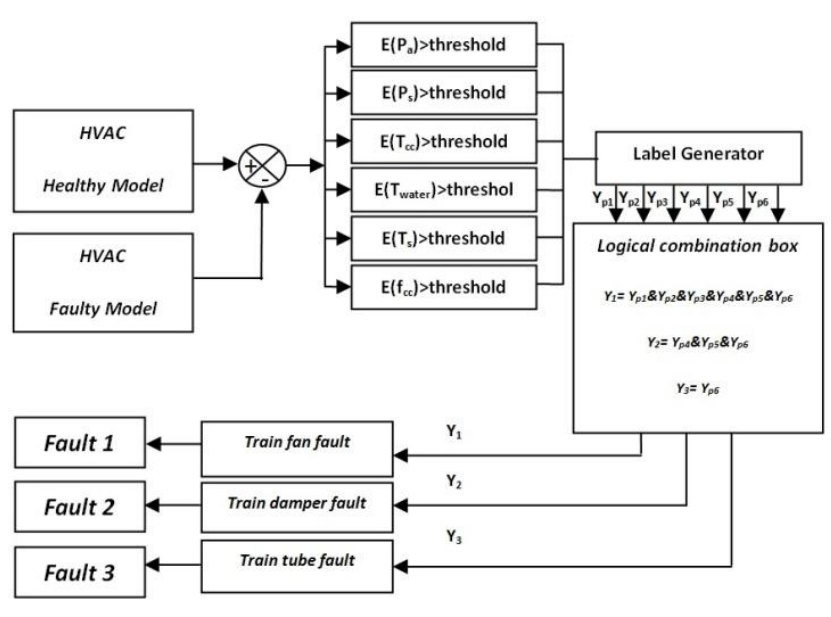

Fig. 8. Schematic of label generation algorithm.

Fig. 8 shows the structure of the label generation algorithm. Here, label of $y$ is set as +1 (non-faulty) when the error is smaller than a given threshold and it is set as -1 (faulty) when the error is bigger than that threshold. Labels including $\mathrm{y}_{\mathrm{p} 1}, \ldots, \mathrm{y}_{\mathrm{pn}}$ are generated with $n$ variables for each fault. For each fault all labels should be combined together in a proper logic to generate one label as one fault needs just one label for training. A combination of labels can be used for generate final label. But for real complex HVAC system, we recommend using a fuzzy logic membership function and some rules to generated final label. In this paper, a specific fault can be happen if all errors of sensitive parameters can passed from their thresholds.

\section{Simulation For FAUlt DeteCtion AND IsOlation}

Since the SVM classifier presented in last section can only be used to deal with two-class case, a multi-layer SVM framework has to be designed for the FDI problem with various faulty conditions. In order to use online SVM classification method to achieve a better isolation performance, three faulty models are used in the isolation section. A four-layer SVM classifier is designed, in which the normal and three different HVAC fault conditions are all taken into consideration. Furthermore, it should be pointed out that other unknown faulty conditions can be placed in the upper layer of the FDI system. The kernel function must be properly selected for SVM classifier in order to achieve high classification accuracy. In general, linear function, polynomial function, radial basis function (RBF), sigmoid function, and Gaussian function can be adopted as the kernel function. In this paper, Gaussian function is used as it has excellent performance in the simulation.

In this research, two tests are conducted systematically. The diagnosis results and corresponding characteristics of the SVM classifiers are shown in Fig. 9, Fig. 10 and Fig. 11. Test 1 is designed to investigate the SVM classifier performance on known incipient faults. The steady-state data is used to build the four-layer SVM classifier: as mentioned in previous section, the data within the threshold under the normal condition indicate fault free, and the data beyond the threshold indicate faults 1 to 3 . For each normal/faulty condition, two days data ( 20 hours data per day between $2 \mathrm{am}$ and $22 \mathrm{pm}$ ) are used. Therefore, a total of 4 times 40 hour samples are collected. Half of the data for each condition are used as the training data, whilst the rest are used as the testing data for fault diagnosis.

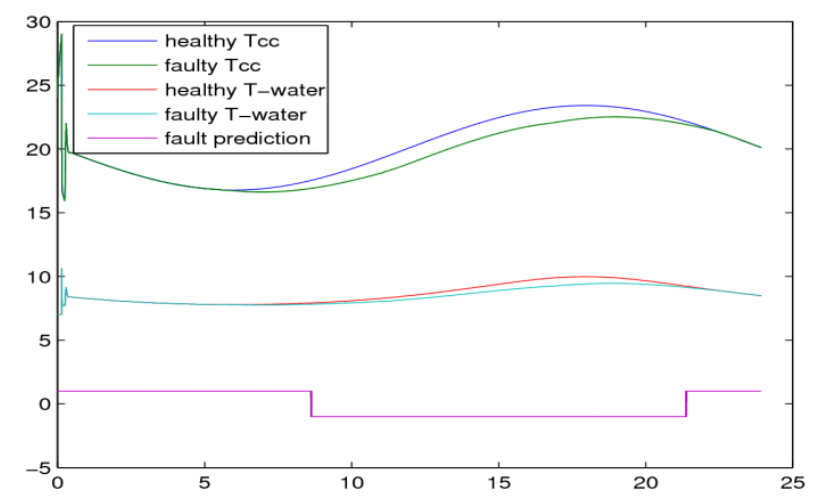

Fig. 9. Graduate known fault detection.

In Fig. 9, the label changes from +1 (non-faulty situation) to -1 (faulty situation) when the fault is detected. For simplicity Fig. 9 only shows two variables when there is fault 1 in the data: the cooling coil temperature and the water outlet temperature. It is clear that the HVAC faults can be diagnosed $100 \%$ by using the SVM classifier for the testing data.

As mentioned earlier, our proposed algorithm is able to detect unknown faults in the sense of semi-unsupervised manner. To testing semi-unsupervised performances, an unknown sudden fault (at time of $9 \mathrm{~h}$ ) combined with previously introduced incipient faults are imposed on system at second test. The detection results are shown in Fig. 10.

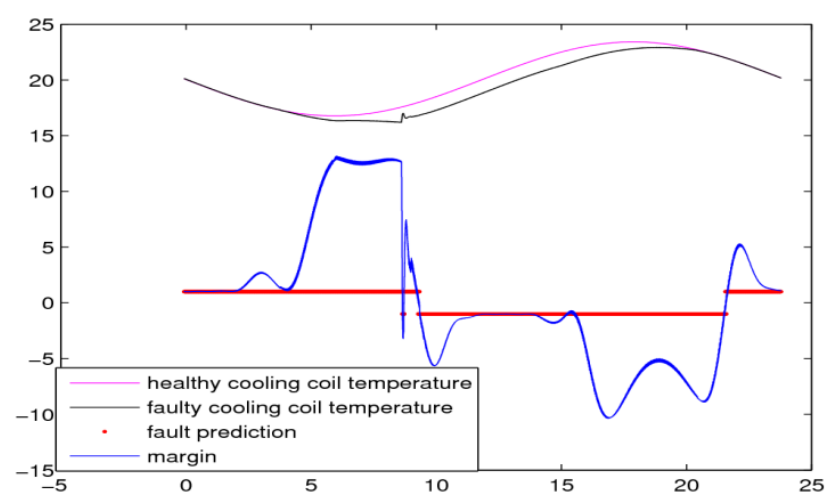

Fig. 10. Sudden unknown fault detection.

It is clearly indicated that the margin changes from high level to low level when detecting incipient fault. This change is dramatic as unknown fault is abrupt type. To efficiently optimizing training process, samples in each normal/faulty condition should be applied. A group containing of maximum faulty training samples is selected, and applied for training. From the Fig. 10, it is found that the designed SVM classifier can identify the HVAC unknown fault accurately. Based on the simulation result, it is found that by using the proposed approach, the unknown faults of HVAC system can also be detected efficiently. 


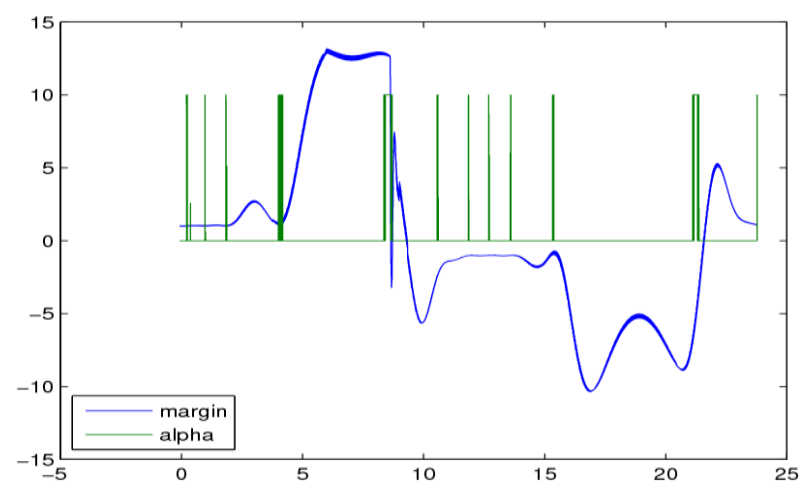

Fig. 11. Training coefficient via margin change.

We can see alpha $\left(\boldsymbol{\alpha}_{i}\right)$ coefficient change with respect to margin change in Fig. 11. As mentioned in previous section, this coefficient should be confine between zero and maximum penalty as shown in this Fig.

\section{CONCLUSION}

This paper focuses on the fault detection and isolation of HVAC system under real time working conditions. An online SVM FDI classifier has been developed which can be trained during the operating of the HVAC system. Different with off line method, the proposed approach can even detect new unknown faults for the training of the classifier in real time. Furthermore, this online approach can more efficiently train the FDI modular by throwing out unnecessary data (leave out vectors) and just used a series of data with high priority regarding to classification. Due to these properties, the proposed algorithm can be implemented in a semi-unsupervised learning frame work. Simulation study indicates that the proposed approach can efficiently detect and isolate typical HVAC faults. In the next step, we will validate the proposed approach by using real experimental data.

\section{REFERENCES}

[1] S. W. Wang, Q. Zhou, and F. Xiao, "A system-level fault detection and diagnosis strategy for HVAC systems involving sensor faults," Energy and Buildings, vol. 42, no. 4, pp. 477-490, 2010.
[2] J. Liang and R. Du, "Model-based fault detection and diagnosis of HVAC systems using support vector machine method," International Journal of Refrigeration, vol. 30, no. 6, pp. 1104-1114, 2007.

[3] T. I. Salsbury and R. C. Diamond, "Fault detection in HVAC systems using model-based feedforward control," Energy and Buildings, vol. 33, no. 4, pp. 403-415, 2001.

[4] Q. Zhou, S. W. Wang, and Z. J. Ma, "A model-based fault detection and diagnosis strategy for HVAC systems," International Journal of Energy Research, vol. 33, no. 10, pp. 903-918, 2009.

[5] K. Zhang, B. Jiang, and P. Shi, "A new approach to observer-based fault-tolerant controller design for takagi-sugeno fuzzy systems with state delay," Circuits Systems and Signal Processing, vol. 28, no. 5, pp 679-697, 2009.

[6] P. S. Kim and E. H. Lee, "A new parity space approach to fault detection for general systems," High Performance Computing and Communications, Proceedings, vol. 37, no. 26, pp. 535-540, 2005

[7] Y. Dote, S. J. Ovaska, and X. Z. Gao, "Fault detection using RBFNand AR-based general parameter methods," 2001 Ieee International Conference on Systems, Man, and Cybernetics, vols. 1-5, pp. 77-80, 2002.

[8] H. Henao, et al., "An improved signal processing-based fault detection technique for induction machine drives," Iecon'03: The 29th Annual Conference of the Ieee Industrial Electronics Society, Proceedings, vols. 1-3, pp. 1386-1389, 2003.

[9] Z. M. Du, X. Q. Jin, and B. Fan, "Fault diagnosis for sensors in HVAC systems using wavelet neural network," Acra 2009: Proceedings of the 4th Asian Conference on Refrigeration and Air-Conditioning, 2009, pp. 409-415.

[10] C. H. Lo, et al., "Fuzzy-genetic algorithm for automatic fault detection in HVAC systems," Applied Soft Computing, vol. 7, no. 2, pp. 554-560, 2007.

[11] B. A. Serrano and M. V. Reyes, "Nonlinear control of a heating, ventilating, and air conditioning system with thermal load estimation," Control Systems Technology, IEEE Transactions on 1999, vol. 7, no. 1 , pp. 56-63, 1999.

[12] B. Tashtoush, M. Molhim, and M. Al-Rousan, "Dynamic model of an HVAC system for control analysis," Energy, vol. 30, no. 10, pp 1729-1745, 2005.

[13] L. Jian and D. Ruxu, "Thermal comfort control based on neural network for HVAC application," in Control Applications, 2005. CCA 2005. Proceedings of 2005 IEEE Conference on. 2005

[14] A. S. Cerqueira, et al., "Power quality events recognition using a SVM-based method," Electric Power Systems Research, 2008, vol. 78 , no. 9, pp. 1546-1552.

[15] G. Cauwenberghs and T. Poggio, "Incremental and decremental support vector machine learning," Advances in Neural Information Processing Systems 13, vol. 13, pp. 409-415, 2001.

[16] N. A. Syed, H. Liu, and K. K. Sung, "Incremental learning with support vector machines," in Proc. Int. Joint Conf. on Artificial Intelligence (IJCAI-99), 1999.

[17] V. Vapnik, "The nature of statistical learning theory," New York: Springer-Verlag, 1995.

[18] A. Kathy and F. Chen, "Offline and online performance analysis, master thesis," Electrical and Computer Science Department of Massachusetts Institute of Technology(MIT), 2007 